\title{
Is the PHY Layer Dead?
}

\author{
M. Dohler ${ }^{1}$, R. W. Heath Jr. ${ }^{2}$, A. Lozano ${ }^{3}$, C. Papadias ${ }^{4}$, R. A. Valenzuela ${ }^{5}$
}

\begin{abstract}
This paper originates from a panel with the above title, held at IEEE VTC Spring 2009, in which the authors took part. The enthusiastic response it received prompted us to discuss for a wider audience whether research at the physical layer (PHY) is still relevant to the field of wireless communications. Using cellular systems as the axis of our exposition, we exemplify areas where $\mathrm{PHY}$ research has indeed hit a performance wall and where any improvements are expected to be marginal. We then discuss whether the research directions taken in the past have always been the right choice and how lessons learned could influence future policy decisions. Several of the raised issues are subsequently discussed in greater details, e.g., the growing divergence between academia and industry. With this argumentation at hand, we identify areas that are either underdeveloped or likely to be of impact in coming years - hence corroborating the relevance and importance of $\mathrm{PHY}$ research.
\end{abstract}

\section{Introduction}

Wireless communication has been the subject of much hype: branded with terms like ubiquitous, pervasive, fundamental, paradigm-shifting, and revolutionary. Cellular communication systems have been the focus of this Broadway play, with the physical layer (PHY) taking the leading role. After decades of thriving advances and exciting developments such as OFDM, turbo codes, LDPC codes, or MIMO, cellular communication systems are becoming mature. Doubts about the viability and relevance of PHY research have naturally arisen, leading to a growing sense that the work in this area is generating diminishing returns and that the discipline may be essentially dead. But is it?

Doubts about the likelihood of great advances in a particular field are frequently expressed in scientific communities and, in fact, they may spur healthy debates; [Smo06], for instance, discusses the negative impact that the hype on string theory had on how funding was distributed within physics communities. Actually, doubts have been cast on research within the PHY before. For example, in a 1971 workshop on coding theory, Ned Weldon famously declared: “coding is dead” [Luc93]. In hindsight, of course, coding was not dead and a number of breakthroughs were still to come.

\footnotetext{
${ }^{1}$ CTTC, Barcelona, Spain

2 The University of Texas at Austin, USA

${ }^{3}$ UPF, Barcelona, Spain

${ }^{4}$ AIT, Athens, Greece

${ }^{5}$ Bell Labs, USA
} 
The aim of this article is to diagnose the condition of PHY research today. This may prove useful in future decisions related to R\&D within the PHY and hopefully positively influence policy makers deciding on international and national project grants, companies' research directions, among others.

To frame the discussion, it is healthy to provide a definition of the PHY to ensure a common understanding. Having its origins in wireline systems, the Open Systems Interconnection (OSI) model formally attributes to the PHY the transmission of bits over a dedicated link with the aid of baseband and radio frequency (RF) processing at the transmitting and receiving ends. In wireless systems, however, links are not cleanly defined in isolation because of the nature of the radio medium and the inevitable interlink interactions. Within the wireless community, we believe the PHY definition should be formally broadened to concern the transmission of bits from transmitters to receivers, encompassing, e.g., multiuser, multicell and relay configurations. This broader definition accommodates issues that are central to the design of wireless systems today and for years to come.

\section{Hitting The Wall}

In 1948, Claude E. Shannon published his impact treatise and put a clear border around the region within which reliable communication is feasible. The notion of capacity was born. At that time, when it was believed that ridding the communication of errors required driving the data rate to zero, this revelation triggered a revolution - and with it an obsession to design systems capable of approaching such capacity as tightly as possible [Mas84]. By 1993 we were within a few dB of the single-link AWGN channel capacity, when the publication of another landmark paper [Ber93] virtually closed this gap.

Today, we have an excellent knowledge of the capacities of most single-link channel settings of interest, with the inclusion of fading and MIMO. Moreover, this understanding is rapidly extending to the point-to-multipoint and multipoint-to-point settings that describe the downlink and the uplink of an individual cell. We also have, by now, transceiver designs that operate increasingly close to these capacities. The importance of these advances cannot be overstated. However, should we continue working on these problems now that the performance limits are essentially within reach?

By contrast, the capacities of other basic settings of interest forming an integral part of modern communication systems are not yet known; examples include simple channels featuring relays or mutual interference, our knowledge of whose exact capacities has hardly advanced for years. Only inner and outer bounds, established long ago in some cases, are available. Yet these bounds may well suffice for most purposes. Is it worth continuing to try to refine this knowledge?

In its relentless progress, the PHY community has developed an extensive set of mathematical tools and has evolved to a high level of proficiency. Can this be transplanted to more sophisticated system problems, e.g., to problems that go beyond the traditional single-link or single-cell settings? 
Hitting the aforementioned performance wall, or approaching it closely enough, the community has accepted the posing and answering of questions that do not relate to exact expressions but rather to bounds and asymptotic operating conditions. For instance, it is natural today to develop mathematical expressions for the cases of the SNR approaching zero or the number of antennas approaching infinity. Is PHY research getting lost in asymptopia?

A more insidious question is whether past improvements in the PHY have really made a dramatic difference in the first place. Martin Cooper, one of the fathers of cellular telephony, observed at some point that the wireless throughput had doubled every 30 months over a period of 104 years, which translates into approximately a million-fold increase since 1957. Breaking down these gains, the main constituents turn out to be a 25 -fold increase from the availability of wider spectrum and an impressive 1600-fold gain due to reduced cell sizes. Only a 5-fold increase is due to all other PHY improvements [Cha08]. Altogether then, is the relatively small improvement associated with a better PHY (smaller cells and wider bandwidths excluded) a satisfactory return on the corresponding research investment over the years? To balance the argumentation, note that, although powerful arguments in principle, smaller cells and wider bandwidths go hand in hand with major increases in capital and operational costs.

Sometimes, the real world and the applications steal the impact of excellent fundamental PHY research. Concerning spectral sensing, for example, [EC1] suggest that many factors will hinder potential uses of sensing techniques to facilitate cognitive or white space radio systems. They hint at the use of more reliable approaches, based either on geolocation or on pilot channels providing spectral usage information, somehow marginalizing much of the research conducted in the area of spectral sensing. Concerning MIMO, [OFC2, HV05] reveal that if all system parameters in a real-world roll-out scenario are taken into account, simple fixed-sector multi-beam antennas are sometimes the best solution in terms of cost-benefit tradeoff. This is further corroborated by the well-known insight that some forms of MIMO are most useful at high SNR, which is a condition rarely seen in many communication systems. How much should $P H Y$ research be conditioned by practical limitations and specific applications?

Notice that we have prominently referred to the notion of capacity, but is that really the metric on which we should be focusing? The danger exists that the obsession with capacity may be obfuscating some of the PHY work.

Zooming out from the details, the overarching question that arises is: looking forward, can PHY research lead to dramatic improvements in communication performance? PHY research might be long lived if we are still far from system-wide fundamental limits.

\section{3. $R$ versus $D$}

Before addressing the questions posed in the previous section, it is useful to examine the roles of the two main players in the creation of PHY technologies: academia, rather associated to R (research), and industry, rather associated to D (development). Coarsely, academia is concerned with theory while industry with development and 
commercialization of technology. With some exceptions, industry and academia have increasingly begun to operate under their own rules and to thus speak different languages.

This disconnection between academia and industry has increased the perception of morbidity of the PHY. Academics largely judge themselves by the theory developed in their papers, on aspects of generality and elegance. They compete in areas like coming up with new mathematical tools or better bounds on performance. Industry is judged by the stock market on its ability to turn a profit. Developing intellectual property and incorporating it into a standard may be more important than ensuring that the standard has the best technology. Both groups are encouraged by self-feedback loops to continue their respective developments, i.e., generating more papers vs. more standards contributions / intellectual property / profits.

The checks and balances provided by feedback between academia and industry are breaking down. For example: why doesn't academia work on PHY problems where there are larger gains? Why doesn't industry leverage academic research to a greater extent to find the best solutions? There is a fundamental communication issue. Academia presents results at technical conferences and industry at standards meetings. The former reads the IEEE Transactions and the latter the Financial Times. The lack of feedback means that industry is not grounding academia about the gains of theory under practical assumptions while academia, with notable exceptions, is not providing theoretical intuition and design guidelines that can assist the development of complex systems.

One way to reinvigorate PHY research is to reconnect academia and industry. There are recent examples of successful interplay between academia and industry. An example is limited-feedback precoding, where channel-state information is quantized at the receiver and conveyed back to the transmitter [Hea08]. The development of limited feedback was inspired by the practical problem of obtaining channel-state information at the transmitter. The Grassmannian manifold, being the academic term underlying this theory, has subsequently been discussed and incorporated into commercial wireless standards including IEEE 802.16e WiMAX and 3GPP LTE. It is important to revive this link and we believe that the steps below could be a starter to improve communication between academia and industry:

- Academia, on one hand, needs to realize that wireless systems are complex. Simulation, a language well understood in the industrial world, plays an important role in their evaluation. Consequently, academic publishing venues should be more supportive of papers with (good) simulations. To make simulation results more credible, authors should be encouraged to maximize reproducibility by describing their simulation methods and parameters in detail, and making their simulation code available via software repositories [Vet09]. The same comments hold for experimentation, which is in essence the only final verification of ideas and simulations. Academia may also help industry not to fall into the trap of Westheimer's Law, i.e. "a few months in the laboratory will save you a few hours in the library” [Moo09].

- Industry, on the other hand, should use academia's expertise more rigorously to tame and, if needed, quell hype around emerging 'unprecedented' technologies. (As an example, the issue of cross-layer design is discussed in Section 4.2. For 
more examples, see [Cox08].) This expertise should equally be queried when pushing for technologies that are ripe. Industry should also provide more insight into physical impairments in the hardware so that academics can develop better models and thus more accurate theory. Last, but not least, industry should make standards and the entire standardization process more accessible. A good starting point would be making contributions easier to search, reducing the time and cost of standardization workshops, and making draft standards freely accessible for research use.

\section{Not Quite PHYnished}

Assuming the communication problem between $\mathrm{R}$ and $\mathrm{D}$ can be solved, the original problem statement on the viability of PHY research remains. We next revisit some of the questions posed in Section 2 to discuss shortcomings but also hint at ways forward.

\subsection{What is left in single-link communication research?}

With the performance limits of most single-link communication settings of interest well characterized and with practical transceiver architectures developed, only incremental improvements are possible for these settings. Future work will have to go beyond these. A viable path for keeping the single-link research alive is to revisit the assumptions that form the foundation of the wall that has been hit:

- Short Codes. Recall the conventional definition of the capacity as the rate that can be achieved with arbitrarily low probability of error with increasing coding block-lengths. The wall has consequently been reached only under certain conditions, such as long block-lengths. In wireless systems, though, there is still a great need for short codes that are powerful yet reasonably simple to decode. It is known, from various error exponents, that good short codes are feasible [Pol10]. Effective short codes would be instrumental, e.g., in latency-constrained applications or for control channels.

- New Coding Paradigms. After the discovery of turbo and LDPC codes, coding theory was again thought by some to be dead. Lateral thinking, though, brought about the concept of rateless or fountain codes, which have special structures that permit decoding upon observing a sufficient number of symbols in any given window. These codes are suitable for broadcast transmission and can be applied as a generalization of hybrid ARQ. Even more recently, polar codes [Ari09] have opened up a new coding avenue where capacity can, not only be approached with affordable complexity (as in turbo or LDPC), but it can be achieved provably in certain channels.

- Impairments. Although the impairments considered in standard single-link wireless settings are almost exclusively fading and AWGN, other impairments are present in real systems caused by implementation at the sub-PHY layer (our name for the unlabeled part of the PHY that employs researchers in circuits, mixed signal, and electromagnetics). Nonlinearities in power amplifiers, a result of power efficiency / cost tradeoffs, can expand the bandwidth of the transmitted signal and create distortions that increase signal decoding complexity. Phase 
noise, present due to imperfect oscillators, creates a multiplicative noise effect that is all too often neglected by theory but is increasingly important in $60-\mathrm{GHz}$ wireless communication systems, for example. Non-Gaussian noise is also present, due for instance to platform noise when wireless chips are implemented close to high-frequency central processors. Some apparent impairments may actually turn out to have beneficial side-effects if treated appropriately (mutual coupling, for example, can be exploited in order to build compact antenna arrays for spatial multiplexing [Alr09]).

Altogether, opportunities for more realistic theory (better defined walls) and more holistic designs (approaching new walls) still abound. However, much of the current research, especially in academia, does not pursue these opportunities but rather keeps insisting on the standard settings where only incremental improvements are possible, at best.

\subsection{Can expertise in single-cell communication be transplanted to broader system problems?}

As outlined earlier, the performance limits of most single-cell communication settings of interest are also known and essentially within reach. To continue achieving large gains in communication system performance, the corresponding expertise must be transplanted to broader settings:

- Interference and Coordination. Rethinking the role of base stations as uncoordinated interferers led to network MIMO, ${ }^{6}$ where the backhaul connection between base stations is used to coordinate their transmissions and thus turn interference into usable signal [Kar06]. Also in the interference front, recent work [Jaf08] studied a high-SNR capacity metric known as degrees-of-freedom, and showed that a new strategy called interference alignment was optimal (in terms of degrees-of-freedom, i.e., in terms of scaling with the SNR in log-scale). Even though its ultimate impact is still unclear, the interference alignment concept is stirring the community and has reinvigorated work on achievable strategies for channels with mutually interfering users, thus far mostly within the confines of the high-SNR regime.

- Relaying. As mentioned in Section 2, the relay channel is one of those basic settings whose fundamental limits remain open. In this case, practical applications have revived interest. In industrial circles, the significant reduction in path loss and shadowing variations with regenerative relays have (re-)introduced such techniques into several cellular standards to mainly yield coverage gains, despite complicating the system architecture. Academic circles, alternatively, have focused on relaying as a tool to provide diversity against fading. Diversity, however, is already broadly available in modern systems [Loz10]. Recent and any future developments, such as the two-way, analog network coding or interferenceaware relaying, hence ought to incorporate path loss and shadowing models to provide a more realistic picture of the potential gains.

- Femtocells. As argued in Section 2, the most effective way of increasing wireless throughput is by shrinking the cells [Cox95]. This has a major cost, however, in

\footnotetext{
${ }^{6}$ In 3GPP parlance, this is termed CoMP (Cooperative MultiPoint transmission and reception).
} 
terms of procurement and maintenance of sites, provision of backhaul access, and network planning. The femtocell concept aims at reaping some of these benefits without most of the costs. Precisely, femtocells are user-installed home base stations that leverage the existing home Internet connection for backhaul. They offload traffic and provide superior indoor coverage with minimal operating expenses on the provider, although planning difficulties remain because of the interference with regular cells and the fact that femtocells cannot be easily synchronized, controlled or coordinated by the system operator [Cha08].

- MIMO Revisited. Intuition about link gains does not always translate to complex systems. In single-link MIMO, under a variety of circumstances the transmitter is better off sending to the receiver as many parallel signals as possible and the capacity then scales with the minimum of the number of transmit and receive antennas. In a cell, however, higher sum-rates are achieved by sending information to different users concurrently. With a large number of users, the downlink sum-capacity then scales with the number of transmit antennas rather than with the minimum of the number of transmit and receive antennas. The story is more convoluted in ad-hoc networks, where transmitting a single signal is preferable in some respects since that leaves the most degrees of freedom for spatial interference cancellation at the receiver [Jin09]. Effectively, in an interference-limited ad-hoc network, multiple antennas are sometimes a better leverage for interference cancellation.

- Extending the Turbo Principle. The philosophy of soft-information iterative exchange that was born with turbo codes has proved since to have a much broader scope of application. Today, one can see its influence in areas as diverse as channel estimation, equalization, multiuser detection and interference cancellation, and even in more distant disciplines such as graph theory or artificial intelligence. Despite the obvious challenges in terms of delay and data blocklength, the potential applicability of this philosophy to broader system contexts is enticing.

There are many opportunities for PHY research to graduate to broader system settings and to continue pushing the boundaries of communication performance. The formulation of new non-equilibrium notions of capacity [And08] and the analysis of scaling laws for ad-hoc networks (see [Gup00] and subsequent extensions, thus far within the confines of asymptopia) are steps in this direction.

In system and network settings, moreover, new research avenues open up, for example in the need for distributed optimization. Techniques such as network MIMO or interference alignment entail collective decisions that must be made on the basis of information gathered locally (e.g., channel conditions). While one may posit a central all-powerful processing point, the reality is that today's networks are evolving towards flat architectures where no such points exist. Thus, these optimizations must be carried out on a manner that is not only distributed, but scalable and robust.

There are also opportunities for research that encompasses both the PHY and higher layers of the protocol stack, which brings us to the theme of cross-layer design. The wireless medium offers tempting prospects, perhaps even needs, to shortcut the protocol 
stack in order to optimize performance. There are some impressive cross-layer results already (e.g., on the use of multiple antennas in ad-hoc networks or the scheduling and coordination of transmissions in cellular systems), but, from a fashionable buzzword that was essential for getting a proposal funded, cross-layer made a rapid transition to hype and then overripe well before all the key problems were actually solved. The bad connotation acquired by cross-layer complicates supporting subsequent research efforts that are now viewed as somewhat obsolete. In addition, some cautionary perspectives are in order. Some authors contend that cross-layer design can conflict with the sound and long-term architectural principles that are necessary for proliferation and longevity [Kum05]. Examples have been provided that show how unintended layer interactions can have undesirable consequences and how unbridled cross-layer implementations can lead to messy designs that stifle further innovation and make maintenance difficult.

\subsection{Is PHY research lost in asymptopia?}

Asymptopia is the land of no limits. In asymptopia, performance metrics are evaluated under assumptions of large or small parameters. In fact, capacity expressions available for the single-link and for other settings are the result of coding theorems that already benefit, as mentioned earlier, from an infinite block-length. Other common PHY parameters explored in asymptopia include the SNR, the number of antennas, and the number of users.

Careful application of asymptotics can make PHY research relevant and can provide intuitive design principles. For example, capacity is achieved at low SNR by peaky signals, which justifies the choice of certain ultrawideband signalling schemes. MIMO communication has also benefited a great deal from asymptotic scaling laws; the highSNR capacity behaviour of MIMO instigated a tremendous amount of research on multiantennas. Scaling laws can often provide more intuition than precise results; exact results on average bit error rate or ergodic capacity eliminate simulation time, but do not always yield as much insight as their asymptotic counterparts. Researchers who are approaching asymptopia should, however, proceed with caution:

- Convergence Rate. When studying limits and scaling laws, the convergence rate is critical yet often overlooked. If the limiting behaviour is not approached with practical choices of parameters, the asymptotes may be mostly anecdotal. At the very least, the range of validity of the results should be verified. For instance, in MIMO Rayleigh-faded channels, capacity expressions asymptotic in the numbers of antennas are accurate even for four-antenna configurations [Big02], which are now practical; under Ricean fading, however, the convergence of the MIMO capacity to the asymptotic expressions is very slow [Loz06, Myth 11].

- Metrics. Depending on the metric of choice, asymptotic analysis is not always appropriate or illuminating. For instance, in a cellular system with a large number of users, the log-log benefits from multiuser diversity are essentially an artifact of the unbounded support of the fading distributions commonly invoked in the models. Further, MIMO capacity scaling laws in SNR are not as relevant in cellular systems because of the low-SNR operating points. As noted above, however, the capacity scaling laws in antennas are reasonable even for fourantenna configurations, which are now practical. 
- Multiple Limits. Special care should also be exercised when taking multiple simultaneous limits. For instance, letting the number of receive antennas in MIMO grow without bound might be incompatible with a low-SNR limiting analysis if each receive antenna is capturing additional power.

Opportunities for applying asymptotic analysis in new systems are clearly present, but researchers should proceed with caution and rigorously justify the applicability of the results.

\subsection{How much should PHY research be conditioned by practical limitations and specific applications?}

Frontier research must always be unhindered. Period. The knowledge thus obtained may turn out to be relevant in unforeseen ways to unforeseen problems in the future, in much the same way as mathematics has evolved often only to find viable applications centuries later.

Research conducted in relation to a specific field, system, or application, should also be largely unhindered in its embryonic stages, when every possibility is still open. At such early stage, the research may be relatively oblivious to details pertaining to specific constraints or to implementational limitations. Models may be simple and need only capture the essence of the problems. As research matures, however, this should progressively change:

- Assumptions. Unreasonable simplifying assumptions should be abandoned. It is not uncommon to find sophisticated mathematical machinery applied to obtain very precise solutions to formulations where the model has been so oversimplified that it only vaguely represents, or even outright misrepresents, the problem under study. If fading is disregarded, for example, the capacity of a complicated wireless setting may be computed precisely - but fading is real and it causes order-ofmagnitude fluctuations in the signal strength. While an initial study may disregard it to focus on other aspects of the problem, at some point fading must be accounted for, even if this takes strenuous and detailed simulations. Other oftenmade simplifying assumptions are: same SNR for all users in a cell, absence of shadowing, negligence of interference, omission of channel code, or no account for capture effects during contention - for all of which elaborate mathematical tools already exist.

- Models. Strongly impacted by underlying assumptions, models have to be refined and elaborated as the research evolves. For instance, diversity schemes that offer substantial gains with flat fading may not yield any gains in wideband channels exhibiting frequency-selective fading; indeed, many authors continue to assume flat fading in their diversity analyses, a premise that is at odds with the operational conditions of many contemporary systems [Loz10]. Another example is adaptivity, a feature of many contemporary wireless systems that often yields surprising performance and reliability gains; link adaptation through feedback fundamentally changes the nature of communication problems - yet, it is often disregarded [Loz10]. 
- Operating Points. It is essential that performance be assessed at the correct operating points, depending on the nature of the system. For example: a code that performs satisfactorily at an error probability of $10^{-6}$, but that performs poorly at $10^{-2}$, might not be useful to a system featuring retransmissions of erroneous data blocks. When appropriate, features of the intended applications should be brought in as well.

- Implementation. Issues pertaining to the actual implementation of PHY algorithms onto hardware platforms should be paid more attention to. Algorithms are needed that can deal with nonidealities, be it at the antennas, with the local oscillators, or in estimating the channel as opposed to assuming it is perfectly known. One of the challenges that opens up is determining which of the impairments can be addressed through baseband processing, leveraging Moore's law, and which ones cannot. Well-designed testbeds / platforms and experiments may be helpful in terms of modelling impairments and testing algorithms.

A tension often arises between tractability and relevance, and in too many occasions researchers choose the path of least resistance. Papers are full of assumptions made 'for convenience' or 'for tractability reasons', but more often than not this is for the convenience of the authors rather than of the problem at hand. As discussed in the previous section, the academic PHY community is often enticed by analytical solutions and, indeed, analytical expressions are extremely useful to identify trends and interplays. However, the scenarios of impact and interest have become increasingly complex over the years, rendering meaningful analytical approaches sometimes fruitless. The value of a solution is largely determined by the model and assumptions used to derive it - yet in many research papers these are poorly justified or even utterly unreasonable. Results that are direct artifacts of the modelling assumptions should be recognized and used to improve those models, rather than simply accepted. At the same time, there is also danger in adding unnecessary facets of reality to the models, as this may smother rather than enlighten and inspire.

A growing challenge for PHY researchers is thus to find the correct level of abstraction for every problem, capturing the necessary features and nuisances while promoting traction and usefulness.

\subsection{Is Capacity really the metric on which we should be focusing?}

Performance metrics other than the capacity or spectral efficiency regions should receive more attention; examples include metrics characterizing the latency, the energy efficiency (e.g., Joules/bit), the complexity (e.g., bits/flop), or the performance for delay-tolerant networks (e.g., bits/day). To buttress the importance of energy efficiency, in particular, one need only compare the enormous increases in throughput and in computational horsepower yielded by Moore's law with the paltry improvement in nominal battery capacity: 3.5\% yearly over the last two decades [Pen09].

Besides alternative metrics to the spectral efficiency, other definitions or extensions thereof may also be considered. The average area spectral efficiency (e.g., bps/ $\mathrm{Hz} / \mathrm{m}^{2}$ ), for instance, is frequently used by operators as a quantifier of both the spectral and spatial efficiency [Alo99]. A still largely unexplored metric is the variance of the area spectral efficiency, which informs of fairness between different parts of the considered region. 
Yet other notions of spectral efficiency are the non-equilibrium ones mentioned earlier in the context of ad-hoc networks [And08] and the transport capacity (e.g., bits-meter) that values both the volume of information and the distance that it is moved over [Gup00].

Also, not all bits are born equal, and it would be desirable to inject the relative importance of different pieces of data into the performance metrics.

\section{Concluding Remarks}

While the PHY may not seem as vibrant an area as it used to be some decades ago, it is clearly an important area with potential for sustained impact. It will continue being at the heart of performance prediction and enhancement, intellectual property protection, as well as cost and complexity reduction. Concerning complexity in particular: baseband computational horsepower has grown by roughly 40 Million since 1957, on account of Moore's law, while the throughput has grown by roughly one million, and thus the complexity burden on the PHY algorithms has loosened. Nonetheless, attention still ought to be paid to complexity as it is still highly relevant in embedded system designs, sensors, and biomedical systems, which cannot leverage all this computational power.

With emphasis on wireless communications, we have tried to identify some compelling issues with the ultimate aim of making everyone in our community, be it a student, academic or industrial researcher, alert to embark onto problems not for the problem's sake but for the solution's sake. We have also tried to highlight areas within the PHY that deserve special attention, where these are generally applicable to all layers of cellular, WLAN, and embedded systems.

We have argued that research should generally embrace more general operating assumptions than those for which analytical tools are available. This means that PHY research should more rigorously incorporate path loss, shadowing, outer channel codes, interference and feedback, among other factors, as these are issues central to any modern wireless communication system and without which any performance quantification lacks credibility. We have also established that the PHY needs to graduate to progressively more complete network settings, with a system-level view. The spotlight should be on systems and even on systems of systems. The research toolbox should include not only analysis but also reproducible simulation and experimentation, as appropriate.

Some other promising areas besides the mentioned network topics are millimetre wave (60 GHz systems); Giga-bit information showers (typically at $\mathrm{THz}$ or modulated onto visible light via light bulbs); non-linear information theory (originating in optical systems); information geometry; PHY layer security; machine-to-machine and embedded systems (sensors, actuators, RFIDs); docitive radios (a cross between cognitive and cooperative radios, i.e., radios that teach other radios [Giu10]); green radios and networks; and truly interdisciplinary research involving, e.g., biology and physics.

A final issue is that, beyond any controversy on its impact, PHY research in past decades helped train people and create a network of skilled engineers and researchers. Research is yet another way of teaching and learning that companies should consider when drafting R\&D budgets. Every thought issued so far in our community has been a piece in this infinite jigsaw of research. 


\section{Acknowledgements}

The authors would like to thank Profs. Ramon Agustí and Oriol Sallent (UPC, Barcelona) for the opportunity of organizing the VTC Spring 2009 panel that led to this paper. Valuable feedback and criticism from a number of colleagues is also gratefully acknowledged: Angeliki Alexiou (University of Piraeus), Jeff Andrews (The University of Texas at Austin), Ezio Biglieri (UPF), Gerhard Fettweis (Technische Universität Dresden), Jerry Foschini (Bell Labs), Stefano Galli (Panasonic), David Gesbert (Eurecom), Andrea Goldsmith (Stanford University), David Goodman (Polytechnic Institute of NYU), Maxime Guillaud (Technische Universität Wien), Lajos Hanzo (University of Southampton), Nihar Jindal (University of Minnesota), Aris Moustakas (University of Athens), Stavros Toumpis (Athens University of Economics and Business), William Webb (OFCOM), and the anonymous reviewers.

\section{References}

[Smo06] L. Smolin, "The Trouble With Physics: The Rise of String Theory, The Fall of a Science and What Comes Next”, Houghton Mifflin Harcourt, 2006.

[Luc93] R. Lucky, "Coding is Dead" in "Lucky Strikes... Again”, IEEE Press, 1993, pp. 243-245.

[Mas84] J. Massey, "Information theory: The Copernican system of communications", IEEE Communications Magazine, Vol. 22, Dec. 1984.

[Ber93] C. Berrou, A. Glavieux, P. Thitimajshima, "Near Shannon Limit ErrorCorrecting Coding and Decoding: Turbo Codes," IEEE Int'l Conf. in Communications (ICC’93), pp. 1064-1070, 1993.

[Cha08] V. Chandrasekhar, J. G. Andrews, A. Gatherer, "Femtocell Networks: a Survey", IEEE Communications Magazine, Vol. 46, pp. 59-67, Sept. 2008.

[EC1] European Commission, Radio Spectrum Policy Group, Report on Cognitive Technologies RSPG09-299, Oct. 14, 2009, online: http://rspg.ec.europa.eu/_documents/documents/meetings/rspg20/rspg09_29 9.pdf

[OFC2] C. Parini, X. Chen, Y. Alfadhl, J. Bigham, I. Llewellyn, L.G. Samuel, L. Ho, B. Collins, "Semi-Smart Antenna Technology Project," Ofcom Contract No. 830000081, July 2006, online: http://www.ofcom.org.uk/research/technology/research/emer_tech/smart

[HV05] H. Huang, R. A. Valenzuela, "Fundamental Simulated Performance of Downlink Fixed Wireless Cellular Networks with Multiple Antennas", Proc. of Personal, Indoor and Mobile Radio Communications (PIMRC'05), Vol. 1, Sept. 2005, pp. 161-165.

[Hea08] D. J. Love, R. W. Heath, Jr., V. K. N. Lau, D. Gesbert, B. Rao, M. Andrews, "An Overview of Limited Feedback in Wireless Communication Systems,” IEEE J. Sel. Areas in Comm., Vol. 26, pp. 1341-1365, Oct. 2008. 
[Vet09] P. Vandewalle, J. Kovacevic, M. Vetterli, "Reproducible Research in Signal Processing - What, why, and how,” IEEE Signal Processing Magazine, Vol. 26, pp. 37-47, 2009.

[Moo09] S. Moore, "Design and Implementation Series IV: Westheimer's Law", IEEE Communications Magazine, Vol. 47, pp. 138-139, Nov. 2009.

[Cox08] D. C. Cox, "Fundamental limitations on increasing data rate in wireless systems", Technology Leaders Forum, IEEE Communications Magazine, Dec. 2008, pp. 16-17.

[Pol10] Y. Polyanskiy, H. V. Poor and S. Verdú, "Channel Coding Rate in the Finite Blocklength Regime," IEEE Trans. Information Theory, vol. 56, no. 5, pp. 2307-2359, May 2010.

[Ari09] E. Arikan, "Channel Polarization: A Method for Constructing CapacityAchieving Codes for Symmetric Binary-Input Memoryless Channels,” IEEE Trans. Inform. Theory, Vol. 55, July 2009, pp. 3051-3073.

[Alr09] O. N. Alrabadi, C. B. Papadias, A. Kalis, R. Prasad, "A universal encoding scheme for MIMO transmission using a single active element for PSK modulation schemes," IEEE Trans. Wireless Communications, Vol. 8, Oct. 2009, pp. 5133-5143.

[Kay06] M. K. Karakayali, G. J. Foschini, R. A. Valenzuela, "Network coordination for spectrally efficient communications in cellular systems," IEEE Wireless Communications, Vol. 13, Aug. 2006, pp. 56-61.

[Jaf08] S. A. Jafar, S. Shamai, "Degrees of Freedom Region for the MIMO X Channel," IEEE Transactions on Information Theory, Vol. 54, Jan. 2008, Pages: 151-170.

[Doh10] M. Dohler, Y. Li, "Cooperative Communications: Hardware, Channel \& PHY,” Wiley \& Sons, Feb. 2010.

[Loz10] A. Lozano, N. Jindal, "Transmit Diversity v. Spatial Multiplexing in Modern MIMO Systems", IEEE Transactions on Wireless Communications, Vol. 9, Jan. 2010, pp. 186-197.

[Cox95] D. C. Cox, "Wireless Personal Communications: What Is It?", IEEE Personal Communications Magazine, Vol. 2, April 1995, pp. 20-35.

[Jin09] N. Jindal, J. G. Andrews, S. Weber, "Rethinking MIMO for Wireless Networks: Linear Throughput Increases with Multiple Receive Antennas," Proc. of the IEEE Int'l Conf. in Communications (ICC'09), June 2009.

[Gup00] P. Gupta, P. R. Kumar, "The capacity of wireless networks," IEEE Trans. Inform. Theory, Vol. 46, March 2000, pp. 388-404.

[And08] J. G. Andrews et. al, "Rethinking Information Theory for Mobile Ad Hoc Networks,” IEEE Communications Magazine, Dec. 2008, pp. 94-101.

[Kum05] V. Kawadia, P. R. Kumar, "A cautionary perspective on cross-layer design”, IEEE Wireless Communications, Vol. 12, No. 1, Feb. 2005, pp. 3-11.

[Big02] E. Biglieri, A. M. Tulino, and G. Taricco, "How far away is infinity? Using asymptotic analyses in multiple antenna systems," Proc. IEEE Int. Symp. on 
Spread Spectrum Techn.and Applications (ISSSTA'02), vol. 1, pp. 1-6, Sep. 2002.

[Loz06] A. Lozano, A. M. Tulino, S. Verdú, "Multiantenna capacity: Myths and realities", Space-Time Wireless Systems: From Array Processing to MIMO Communications, Cambridge University Press, 2006.

[Pen09] K. Pentikousis, "In search of energy-efficient mobile networking," IEEE Communications Magazine, Vol. 48, No. 1, Jan. 2010, pp. 95-103.

[Alo99] M.-S. Alouini and A. J. Goldsmith, "Area Spectral Efficiency of Cellular Mobile Radio Systems," IEEE Trans. Vehicular Technology, Vol. 48, No. 4, July 1999, pp. 1047-1066.

[Giu10] L. Giupponi, A. Galindo-Serrano, P. Blasco and Mischa Dohler, "Docitive Networks - An Emerging Paradigm for Dynamic Spectrum Management," IEEE Wireless Communications Magazine, Special Issue on Dynamic Spectrum Management, to be published. 